\title{
JOHN V. MURRA, BIBLIOGRAFÍA
}

\author{
David Block ${ }^{1}$
}

La obra erudita de John Murra se caracteriza por su minuciosa atención bibliográfica. Por muchos años Murra elaboró la sección "EthnohistorySouth America" del Handbook of Latin American Studies. Además ofreció a sus lectores un comentario actualizado sobre las fuentes de estudios andinos (por ejemplo, ver 1968b). A John Murra debemos el mismo cuidado en compilar su propia bibliografía.

Esta contribución no es ni será la única que intenta catalogar la fecunda obra de Murra. Al comenzar el proyecto, tuve la ventaja de organizar su archivo personal que contiene ejemplares, a veces hasta varias docenas, de sus numerosas publicaciones. Ni puedo dejar de reconocer la importancia de bibliografías previamente editadas, con mención especial a la "Bibliografía de John Murra", que se publicó en Lima, en Nispa Ninchis (Castro et al. 2000, pp. 55-64). La tarea formidable de esta bibliografía es que identifica las distintas versiones de los ensayos, algunos con vidas largas. Por citar solo un ejemplo, el ensayo clásico "La función del tejido..." primero ofrecido como ponencia en 1958, se reeditó siete veces en Estados Unidos, Perú y Chile, y por última vez en 2002.

Las setenta y dos referencias que se listan a continuación no pretenden ser globales. Sólo se apuntan las obras investigativas escritas o editadas por Murra. En otra bibliografía, que saldrá en el anuario Andean Past, anotamos casi cuatro decenas de recensiones, entrevistas, homenajes y artículos de prensa periódica que reflejan otras facetas del hombre. Y como lo demuestra la reedición del ensayo sobre Julio Tello en una colección recién salida (ver 1982a), la bibliografia de Murra tal vez nunca estará al día. Aunque no sea completa, esta compilación ilumina una característica fundamental de la obra murriana: el deseo del autor de asegurar su accesibilidad a través del tiempo. Murra también insistió que lectores latinoamericanos, sus verdaderos destinatarios, tuvieran acceso a ediciones de bajo costo.

Ojalá que esta bibliografía ofrezca un derrotero fiel a los que quieren identificar las publicaciones de John V. Murra y seguir su compleja historia editorial.

\section{Bibliografía}

Collier, Donald y John V. Murra con Sharat K. Roy 1943 Survey and Excavations in Southern Ecuador. Field Museum of Natural History Publication 528, Chicago. También publicada en español como Reconocimientos y excavaciones en el sur del Ecuador. Centro de Estudios Históricos y Geográficos de Cuenca, Cuenca (1982); y como Reconocimientos y excavaciones en el austro ecuatoriano (2007). Casa de la Cultura Ecuatoriana, Núcleo del Azuay, Cuenca.

Murra, John V.

1946 The historic tribes of Ecuador. En Handbook of South American Indians, editado por Julian H. Steward, Volumen 2, pp. 785-821. Government Printing Office, Washington, D.C., U.S.

1948 The Cayapa and the Colorado. En Handbook of South American Indians, editado por Julian H. Steward, Volumen 4, pp. 277-291. Government Printing Office, Washington, D.C., U.S.
Murra, John V., Robert M. Hankin y Fred Holling 1951 The Soviet Linguistic Controversy: Translated from the Soviet Press. King's Crown Press. (Columbia University Slavic Studies), New York.

1956 The Economic Organization of the Inca State. Ph.D. dissertation, University of Chicago.

1958a On Inca political structure. En Systems of Political Control and Bureaucracy in Human Societies, editado por Verne F. Ray, pp. 30-41. American Ethnological Society, Seattle. Reimpreso como On Inca Political Structure. Bobbs-Merrill Reprint Series in the Social Sciences, A-169 (1958). Indianapolis: Bobbs-Merrill; también publicado en Comparative Political Systems: Studies in the Politics of Pre-industrial Societies, editado por Ronald Cohen y John Middleton, pp. 339-353 (1967). University of Texas Press, Austin. Una versión en español: En torno a la estructura política de los inka, apareció en Murra (1975a), pp. 23-43. 
1958b La función del tejido en varios contextos sociales y políticos. Actas y Trabajos del Segundo Congreso de Historia del Perú, Volumen 2, pp. 215-240. Una versión en inglés fue publicada como Cloth and its functions in the Inca State. American Anthropologist 64(3):710-728 (1962). Una versión en español fue publicada en Wayna 2:15-37 (1970) titulada: La función del tejido en varios contextos sociales en el estado Inca; en Murra (1975a), pp. 145-170; en 100 años de Arqueología en el Perú, editado por Rogger Ravines, pp. 583-608 (1970). Instituto de Estudios Peruanos, Petróleos del Perú edition, Lima; en Arte Mayor de los Andes: Museo Chileno de Arte Precolombino, de Paulina Brugnoli y Soledad Hoces, editado por Julie Palma, con fotografías de Fernando Maldonado, y traducido por Cecilia Contreras y Barbara Caces, pp. 9-19 (1989). El Museo, Santiago de Chile; y en Murra (2002a), pp. 153-170. Murra updated the English-language version for Cloth and Human Experience, editado por Annette B. Weiner y Jane Schneider, pp. 275-302 (1989). Smithsonian Institution Press, Washington, D.C.

1960 Rite and crop in the Inca State. En Culture in History: Essays in Honor of Paul Radin, editado por Stanley Diamond, pp. 393-407. Published for Brandeis University by Columbia University Press, New York. Una versión revisada fue publicada en Peoples and Cultures of South America, editado por Daniel Gross, pp. $377-389$ (1973). Doubleday and Natural History Press, Garden City, New York. Una versión en español: La papa, el maíz y los ritos agrícolas del Tawantinsuyu, fue publicada en Amaru 8:58-62 (1968); en Murra (1975a), pp. 45-58; en Cosmos, Hombre y Sacralidad: Lecturas dirigidas de Antropología Religiosa, editado por Marco Vinicio Rueda y Segundo Moreno Yáñez, pp. 181-193. (1995) Departamento de Antropología PUCE y Ediciones Abya Yala, Quito; y en Murra (2002a), pp. 143-152.

1961a Guamán Poma de Ayala: A Seventeenth-Century Indian's account of Andean civilization. Natural History 70(7):35-46 y 70(8):52-63. Una versión en español fue publicada en Murra (2002a), pp. 375-425.

1961b Social structural and economic themes in Andean ethnohistory. Anthropological Quarterly 34(2):47-59. La versión en español: Temas de estructura social y económica en la etnohistoria y el antiguo folklore andino, fue publicada en Folklore Americano 10:226-238 (1962); y en La Etnohistoria en Mesoamérica y los Andes, editada por Juan Manuel Pérez y José Antonio Pérez Gollán, pp. 95-111 (1987). Instituto Nacional de Antropología e Historia, México.

1962 An archaeological "restudy" of an Andean ethnohistorical account. American Antiquity 28(1):1-4.

1964a Una apreciación etnológica de la visita. En Visita hecha a la Provincia de Chucuito por Garci Diez de San Miguel en el año 1567 por Garci Diez de San Miguel, transcrita por Waldemar Espinoza Soriano, pp. 421-444 (1964). Casa de la Cultura del Perú, Lima.

1964b Rebaños y pastores en la economía del Tawantinsuyu. Revista Peruana de Cultura 2:3-28. También publicada en Murra (1975a), pp. 117-144; y en Murra (2002a), pp. 309327. Una versión en inglés, Herds and herders in the Inca State, fue publicada en Man, Culture and Animals: The Role of Animals in Human Ecological Adjustments, editada por Anthony Leeds y A. P. Vayda, pp. 185-216 (1965). American Association for the Advancement of Science, Washington. 1966a El Instituto de Investigaciones Andinas y sus estudios en Huánuco, 1963-1966. Antropología, pp. 7-21 (Universidad Nacional Hermilio Valdizán, Huánuco, Perú).

1966b New data on retainer and servile populations in Tawantinsuyu. En Proceedings: International Congress of Americanists 36 (1964). Sevilla, pp. 35-45. Una versión en español, Nueva información sobre las poblaciones yana, fue publicada en Murra (1975a), pp. 225-242; en Murra (2002a), pp. 328-340; y como Nuevos datos sobre las poblaciones yana en el Tawantinsuyo. Antropología Andina 1-2:13-33 (Cusco, 1976).

Thompson, Donald E. y John V. Murra

1966c The Inca Bridges in the Huánuco Region American Antiquity 31(5) Part 1:632-639. También publicado en Peruvian Archaeology: Selected Readings, editado por John Howland Rowe y Dorothy Menzel, pp. 235-242. Peek Publications, Palo Alto, California. Una versión en español, Puentes incaicos en la región de Huánuco Pampa, fue publicada en Antropología, pp. 79-94 (1966). (Universidad Nacional Hermilio Valdizán, Facultad de Letras y Educación, Cuadernos de Investigación 1, Huánuco, Perú).

1967a La visita de los Chupauchu como fuente etnológica. En Ortiz de Zúñiga (1967c), Volumen 1, pp. 381-406.

1967b Ethnohistory: South America. Handbook of Latin American Studies 29:200-201.

Ortiz de Zúñiga, Iñigo

1967c Visita de la Provincia de León de Huánuco en 1562, editado por John V. Murra, 2v. (1967, 1972) Huánuco, Perú: Universidad Nacional Hermilio Valdizán, Facultad de Letras y Educación. Series: Documentos para la historia y etnología de Huánuco y la selva central, 1, 2.

1968a An Aymara Kingdom in 1567. Ethnohistory 15(2):115151. Una versión en español fue publicada como Un Reino Aymara en 1567. Pumapunku 6:87-93 (1972); 9:31-49 (1975); en Murra (1975a), pp. 193-224; y en Murra (2002a), pp. 183-207.

1968b Perspectivas y actuales investigaciones de la etnología andina. Revista del Museo Nacional 35:124-158 (1967-1968); y en Murra (2002a), pp. 445-470; también publicada con el título: Las investigaciones en etnohistoria andina y sus posibilidades en el futuro, en Murra (1975a), pp. 275-312; y en La Etnohistoria en Mesoamérica y los Andes México, editado por Juan Manuel Pérez Zevallos y José Antonio Pérez Gollán, pp. 113-158 (1987). Instituto Nacional de Antropología e Historia, México, D.F. Una versión en inglés fue publicada como Current research and prospects in Andean ethnohistory. Latin American Research Review 5(1):3-36 (1970).

1970a Información etnológica e histórica adicional sobre el reino Lupaqa. Historia y Cultura (Lima) 4:49-61.

1970b Ethnohistory: South America. Handbook of Latin American Studies 32:103-105.

1972a El Control vertical de un máximo de pisos ecológicos en la economía de las sociedades andinas. En Visita de la Provincia de León de Huánuco en 1562, editado por John V. Murra, Volumen 2, Visita de los Yacha y Mitmaqkuna Cuzque, pp. 427-476. También publicada como monografía por la Universidad Nacional Autónoma de México (UNAM) Iztapalapa, División de Ciencias Sociales, en Murra (1975a), pp. 59-115; y con correcciones en Murra (2002a), pp. 85-125. 
1972b Ethnohistory: South America. Handbook of Latin American Studies 34:129-130.

1974a Las etnocategorías de un khipu estatal. En Homenaje a Gonzalo Aguirre Beltrán, editado por Gonzalo Aguirre Beltrán. Volumen 2, pp. 167-176 (1974). Instituto Indigenista Interamericano, México. Republicado en Murra (1975a), pp. 243-254; y en Murra (2002a), pp. 248-260.

1974b Ethnohistory: South America. Handbook of Latin American Studies 38:108-109.

1975a Formaciones Económicas y Políticas del Mundo Andino. Instituto de Estudios Peruanos, Lima. This is a reprinting of 12 essays, originally published between 1958 and 1970. Each gives a genealogy of publication. There is an Italian language edition, Formazioni economiche e politiche nel mondo andino: Saggi di etnostoria (1980). Giulio Einaudi, Torino.

1975b Las autoridades étnicas tradicionales en el Alto Huallaga. Publicado en pp. 171-192 en Murra (1975a). Originalmente publicada como Part II en Murra (1967a) pp. 386-398.

1976a Los límites y las limitaciones del "Archipiélago Vertical" en los Andes. En Homenaje al Dr. Gustavo Le Paige, SJ., editado por José María Casassas, pp. 141-146. Universidad del Norte, Antofagasta, Chile. También publicado en Ensayos sobre el Desarrollo Económico de México y América Latina (1500-1975), editado por Enrique Florescano (1979), pp. 193-198. Fondo de Cultura Económica, México; y en Murra (2002a), pp. 126-131. Una versión en inglés, The limits and limitations of the "Vertical Archipelago" in the Andes, fue publicada en Andean Ecology and Civilization: An Interdisciplinary Perspective on Andean Ecological Complementarity, editado por Shozo Masuda, Izumi Shimada y Craig Morris, pp. 15-20 (1985). University of Tokyo Press, Tokyo.

1976b American anthropology, The early years. West Pub. Co., St. Paul (Proceedings of the American Ethnological Society, 1974).

Murra, John V. y Craig Morris

1976c Dynastic oral tradition, administrative records and archaeology in the Andes. World Archaeology 7(3):269-279.

1978a La Organización Económica del Estado Inca. México: Siglo Veintiuno, América Nuestra series 11. This Work appears in four subsequent editions by Siglo Veintiuno, as well as in an English-language edition, The Economic Organization of the Inka State. Greenwich, Connecticut: JAI Press (1980), and as a Rumanian-language edition, Civilizatie inca: organizarea economică statuli incas, traducido por Ata Iosifescu (1987). Editura Stiintifică si Enciclopedică, Bucuresti.

1978b Aymara Lords and their European agents in Potosí. Nova America 1:231-243. Una versión en español fue publicada como La correspondencia entre un "Capitán de la Mita" y su apoderado en Potosí. Historia y Cultura (Lima) 3:45-48 (1978); y en Murra (2002a), pp. 223-234.

1978c La guerre et les rebellions dans l'expansion de l'État Inka. En Murra et al. Anthropologie historique des sociétates andines, Anales (33)5-6, pp. 927-935. Una versión en inglés, The expansion of the Inka State: Armies, war, and rebellions, fue publicada en Murra et al. (1986), pp. 49-58. Una versión en español, La expansión del estado inka: ejércitos, guerras y rebeliones, fue publicada en Murra (2002a), pp. 57-66. 1978d Los olleros del Inka: hacia una historia y arqueología del Qollasuyo. En Historia, Problema y Promesa, edited by Francisco Miro Quesada C., Franklin Pease G. Y., y David Sobrevilla A., Volumen 1, pp. 415-423. Universidad Pontificia Católica del Perú, Fondo Editorial, Lima; también publicada en Murra (1983b); y en Murra (2002a), pp. 287-293.

1978e Ethnohistory: South America. Handbook of Latin American Studies 40:77-78.

1978 f Introduction. En Deep Rivers de José María Arguedas, traducido por Frances Horning Barraclough, pp. ix-Xv. University of Texas Press, Austin.

Revel, Jacques, John V. Murra y Nathan Wachtel, editores 1978g Anthropologie historique des sociétés andines. Annales 33(5-6), special issue. Publicada en una edición en inglés como Anthropological History of Andean Polities, editado por John V. Murra, Nathan Wachtel y Jacques Revel, Murra (1986).

1979 Derechos a las tierras en el Tawantinsuyu. Revista de la Universidad Complutense. Special issue entitled Economía y sociedad en los Andes y Mesoamérica. [unnumbered], pp. 273287. También publicado en Revista del Museo Inka (Cuzco) 25:103-117 (1995); y en Dos Décadas de Investigación en Historia Económica Comparada en América Latina, editado por Margarita Menegus Bornemann, Antonio Ibarra, Juan Manuel Pérez Cevallos y Jorge Silva (1999), pp. 97-111. Colegio de México, Centro de Estudios Históricos; Centro de Investigaciones y Estudios Superiores en Antropología Social; Instituto doctor José María Luis Mora; y Centro de Estudios Sobre la Universidad, UNAM, México, D.F.; y en Murra (2002a), pp. 294-307.

1980a Ethnohistory: South America. Handbook of Latin American Studies 42:135-137.

Guaman Poma de Ayala, Felipe

1980b El Primer Nueva Crónica y Buen Gobierno, editado por John V. Murra y Rolena Adorno. Quechua translations by Jorge Urioste. Colección América Nuestra 31. 3 volúmenes: Siglo XXI, México. Reprinted 1988, 1992. Another edition published in Madrid by Historia 16 in its Crónicas de América series (1987); facsimile CD-ROM of manuscript GKS $22324^{\circ}$, Copenhagen: Royal Library of Denmark, n.d. and on-line facsimile (http://www.kb.dk/permalink/2006/ poma/1189/en/text/, consulted 6 March 2009) supervised by Ivan Boserup with corrected transcription by John V. Murra and Rolena Adorno and Quechua translations by Jorge L. Urioste.

1982a Dimensión internacional de Julio C. Tello. La dimensión internacional de la obra de Julio C. Tello. Histórica 7:53-63 (1982). A footnote, p. 53, states "La revista solo publicó una primera parte, la segunda fue añadida a una publicación privada hecha por el autor" ("The journal only published a first part; the second was added to a private publication by the author"). This has not been located. An English language version, The International Relevance of Julio C. Tello, appears in The Life and Writings of Julio C. Tello, edited by Richard L. Burger. Iowa City: University of Iowa Press, 2009, pp. 55-64.

1982b The mit'a obligations of ethnic groups to the Inka State. En The Inca and Aztec States, 1400-1800, editado por George A. Collier, Renato I. Rosaldo y John D. Wirth, pp. 237-262. Academic Press, New York. Una versión en español, La mit'a al Tawantinsuyu: prestaciones de los grupos 
étnicos, fue publicada en Chungara 10:77-94 (1983); y en Murra (2002a), pp. 261-286.

1982c El tráfico de mullo en la costa del Pacífico. En Primer Simposio de Correlaciones Antropológicas Andino-Mesoamericano, 25-31 de Julio de 1971, Salinas Ecuador, editado por Jorge Marcos G. y Presley Norton, pp. 265-273. Escuela Superior Politécnica del Litoral, Guayaquil. También publicada en Murra (1975), pp. 255-267; y en Murra (2002a), pp. 171-179; y en Reconocimientos y Excavaciones en el Austro Ecuatoriano (see Collier y Murra 1943), pp. 403-418 (2007).

1982d Ethnohistory: South America. Handbook of Latin American Studies 44:102-103.

1983a José María Arguedas, dos imágenes. Revista IberoAmericana 122:43-54.

1983b Los Olleros del Inka: Hacia una Historia y Arqueología del Qollasuyo. Centro de Investigaciones Históricas, La Paz.

1984a Andean societies. En Annual Review of Anthropology 13:119-141.

1984b The cultural future of the Andean majority. En The Prospects for Plural Societies, editado por David MayburyLewis, pp. 30-38. American Ethnological Society, Washington, D.C.

1985a Andean Societies before 1532. En The Cambridge History of Latin America, editado por Leslie Bethell, Volumen 1, pp. 59-90. Cambridge University Press, Cambridge and New York.

1985b Andean Societies before 1532 (bibliographic essay). En The Cambridge History of Latin America, editado por Leslie Bethell, Volumen 11,pp. 15-18. Cambridge University Press, Cambridge and New York.

1985c "El archipiélago vertical” Revisited. En Andean Ecology and Civilization: An Interdisciplinary Perspective on Andean Ecological Complementarity, editado por Shozo Masuda, Izumi Shimada y Craig Morris, pp. 3-14. University of Tokyo Press, Tokyo. Una versión en español, El archipiélago vertical: Once años después, fue publicada en Murra (2002a), pp. 132-139.

1986a Notes on Pre-Colombian cultivation of coca leaf. En Coca and Cocaine: Effects on People and Policy in Latin America; Proceedings of the Conference, The Coca Leaf and Its Derivatives $\tilde{n}$ Biology, Society, and Policy Sponsored by the Latin American Studies Program (LASP), Cornell University, April 25-26, 1985, editado por Deborah Pacini y Christine Franquemont, pp. 49-52. Cambridge, Mass: Cultural Survival and Ithaca, New York: LASP.

1986b Le difficile accouchement d'une histoire andine. En Economies méditerranéennes: équilibres et intercommunications, XIIIe-XIXe siècles. (Actes du IIe Colloque International d'Histoire), Volumen 3, pp. 309-313. Centre de Recherches Néohelléniques de la Fondation Nationale de la Recherche Scientifique, Athènes.

Murra, John V. y Nathan Wachtel

1978c Présentation. Anthropologie historique des sociétés andines. Anales (33)5-6:889-894. Una versión en inglés, Introduction, fue publicada en Murra et al. (1986), pp. 1-9.

Murra, John V., Nathan Wachtel y Jacques Revel

1986d Anthropological History of Andean Polities. Cambridge University Press, Cambridge and New York and Editions de la Maison des Sciences de l'Homme, Paris.
1987 La etnohistoria. En La Etnohistoria en Mesoamérica y los Andes, editado por Juan Manuel Pérez Zevallos y José Antonio Pérez Gollán, pp. 159-175. Instituto Nacional de Antropología e Historia, México.

Condarco Morales, Ramiro y John V. Murra

1987b La teoría de la complementariedad vertical ecosimbiótica. Hisbol. Series Breve Biblioteca del Bolsillo 2, La Paz. Contains Murra's essays, El control vertical de un máximo de pisos ecológicos en la economía de las sociedades andinas, pp. 29-85 (Murra 1967a) y El 'Archipiélago vertical' revisitado, pp. 87-104 (Murra 1985c).

1988a El Aymara libre de ayer. En Raíces de América: El mundo Aymara, editado por Xavier Albó, pp. 51-74. Alianza Editorial, Madrid.

1988b El Doctor Barros de San Millán, defensor de los "señores naturales" de los Andes. En Actas del IV Congreso Internacional de Etnohistoria. Lima: Universidad Pontificia Católica del Perú, Volumen 2, pp. 359-377. También publicado como El Doctor Barros de San Millán: Defensor de los "Señores Naturales" en los Andes. Servei de Publicacions de la Universitat de Barcelona, Barcelona, 1993; y en (Murra 2002a), pp. 426-438.

1989 High altitude Andean societies and their economies. En Geographic Perspectives in History, editado por Eugene D. Genovese y Leonard Hochberg, pp. 205-214. B. Blackwell, Oxford and New York.

1991a Le débat sur l'avenir des Andes en 1562. Cultures et Sociétés: Andes et Méso-Amérique: Mélanges en homage à Pierre Duviols, editado por Pierre Duviols y Raquel Thiercelin, Volumen 2, pp. 626-632. Université de Provence, Service des Publications, Aix-en-Provence.

1991b "Nos hazen much ventaja": The Early European perception of Andean achievement. En Transatlantic Encounters: Europeans and Andeans in the Sixteenth Century, editado por Kenneth J. Andrien y Rolena Adorno, pp. 73-89. University of California Press, Berkeley. Una versión en español, Nos hacen mucha ventaja: percepción europea temprana de los logros andinos, fue publicada en Semillas de Industria, Transformaciones de la Tecnología Andina en las Américas, editado por Mario Ruz, pp. 19-35 (1994). Ciesas, México and Smithsonian Institution, Washington, D.C.; y en Murra (2002a), pp. 25-40.

Murra, John V., editor

1991c Visita de los valles de Sonqo en las yunka de coca de La Paz (1568-1570). Madrid: Instituto de Cooperación Iberoamericana; Quinto Centenario; Instituto de Estudios Fiscales. Includes essay, Introducción al estudio histórico del cultivo de la hoja de la coca [Exythroxylon coca] en los Andes, pp. 565-581 which is subsequently published in Murra (2002a) pp. 359-372.

1994 John Hyslop 1945-1993. Andean Past 4, pp. 1-7.

1995 Did tribute and markets prevail in the Andes before the European invasion? En Ethnicity, Markets and Migration in the Andes, editado por Brooke Larson y Olivia Harris, pp. 57-72. Duke University Press, Durham, North Carolina. Una versión en español, ¿Existeron el tributo y los mercados en los Andes antes de la invasion española? fue publicada en Arqueología, Antropología, e Historia en los Andes: Homenaje a María Rostworowski, editado por Rafael Varón Gabai y Javier Flores Espinoza, pp. 737-748 (1997). Instituto 
de Estudios Peruanos and Banco Central de Reserva del Perú, Lima; también publicada en Murra (2002a), pp. 237-247. Murra, John V. y Mercedes López-Baralt, editores

1996 Las Cartas de Arguedas. Universidad Pontificia Católica del Perú, Lima. Segunda edición (1998).

1997 Litigation over the rights of "Natural Lords" in Early Colonial courts in the Andes. En Native Traditions in the Postconquest World: A Symposium at Dumbarton Oaks, editado por Elizabeth Hill Boone y Tom Cummins, pp. 55-62. Dumbarton Oaks Research Library and Collection, Washington, D.C. Una versión en español, Litigio sobre los derechos de los "señores naturales" en las primeras cortes coloniales en los Andes, fue publicada en Historias 49:101-105 (2001).
1999 El Tawantinsuyu. En Historia General de América Latina, editado por Federico Mayor y Germán Damas, Volumen 1, pp. 481-484. Editorial Trotta, Madrid y Ediciones Unesco, Paris. También publicada en (Murra 2002a), pp. 67-82 además mapa plegable.

2002a El Mundo Andino: Población, Medio Ambiente y Economía. Pontificia Universidad Católica del Perú, Fondo Editorial e Instituto de Estudios Peruanos (Historia Andina 24), Lima.

2002b Barros de San Millán. En Diccionario Histórico de Bolivia, editado por Josep M. Barnadas, Volumen 1, pp. 272-274. Grupo de Estudios Históricos, Sucre. 
